\title{
Differentiation between Tuberculous and Pyogenic Spondylodiscitis: The Role of the Anterior Meningovertebral Ligament in Patients with Anterior Epidural Abscess
}

\author{
(D) S.B. Strauss, (D) S.R. Gordon, (D). Burns, (D).A. Bello, and (D) S.E. Slasky
}

\begin{abstract}
BACKGROUND AND PURPOSE: Differentiation between tuberculous and pyogenic spondylodiscitis is a diagnostic challenge because imaging often does not reliably distinguish the 2 entities and percutaneous biopsies are often culture-negative. The purpose of this study was to determine whether violation of the anterior meningovertebral ligament in the setting of anterior epidural abscess discriminates between these entities.

MATERIALS AND METHODS: This was a retrospective cohort study of all patients with acid-fast bacillus testing and anterior epidural abscess diagnosed on spinal MR imaging between May 2014 and September 2019, with a final diagnosis of tuberculous or pyogenic spondylodiscitis. Six cases of tuberculous spondylodiscitis (mean age, 45.5 years; 80\% male) and 35 cases of pyogenic spondylodiscitis were evaluated (mean age, 56.6 years; 49\% male). Demographic characteristics were recorded. Cases were assessed for anterior meningovertebral ligament destruction on MR imaging, as demonstrated by the shape of the epidural collection. Segmental location of the infection was also assessed. Independent 2 -sample $t$ tests and $\chi^{2}$ tests of independence were performed to evaluate the significance of the difference between the groups.
\end{abstract}

RESULTS: Five of $6(83.3 \%)$ cases of tuberculous epidural abscess had an intact anterior meningovertebral ligament, and $0 / 35$ cases of pyogenic epidural abscess demonstrated an intact ligament $(P<.001)$. The presence of an intact anterior meningovertebral ligament had $83.3 \%$ sensitivity and $100 \%$ specificity for tuberculous spondylodiscitis, a $100 \%$ positive predictive value, and a $97.2 \%$ negative predictive value.

CONCLUSIONS: The presence of an intact anterior meningovertebral ligament has high sensitivity and specificity for tuberculous spondylodiscitis-associated epidural abscess, though these results should be validated in a larger sample.

ABBREVIATIONS: TB = tuberculosis; $\mathrm{CSF}=$ cerebrospinal fluid

nfectious spondylodiscitis is as infection of the vertebral body and intervertebral disc and can be classified as either granulomatous (eg, mycobacterial and fungal), pyogenic (other bacterial organisms), or parasitic depending on responsible pathogen and the host response. ${ }^{1}$ There may be associated paraspinal soft-tissue infection or epidural abscess, though this is not required for a diagnosis of infectious spondylodiscitis. Staphylococcus aureus is the causative organism in $>75 \%$ of cases of bacterial spondylodiscitis, ${ }^{2}$ resulting from either hematogenous spread or direct

Received September 9, 2019; accepted after revision November 3.

From the Department of Radiology (S.B.S.), Weill Cornell Medical Center, New York, New York; and Department of Radiology (S.R.G., J.B., J.A.B., S.E.S.), Montefiore Medical Center, Albert Einstein College of Medicine, Bronx, New York.

Scientific abstract previously presented at: Annual Meeting of the American Society of Neuroradiology, May 18-23, 2019; Boston, Massachusetts.

Please address correspondence to Shira E. Slasky, MD, Montefiore Medical Center, Department of Radiology, 111 East 210th St, Bronx, NY 10467; e-mail:

sslasky@montefiore.org; @ShiraSlaskyMD

http://dx.doi.org/10.3174/ajnr.A6370 extension from trauma or adjacent infection. ${ }^{3}$ Granulomatous spondylodiscitis, specifically tuberculous (TB) spondylodiscitis, is less common than pyogenic spondylodiscitis, with an estimated incidence of only $0.05 / 100,000$ in the United States, ${ }^{4}$ though the incidence is higher in endemic regions. ${ }^{5}$ Differentiation of pyogenic and tuberculous spondylodiscitis is critical because early, targeted treatment can decrease mortality and long-term neurologic morbidity.

The posterior longitudinal ligament is apposed to the posterior vertebral body with direct fixation to the outer annulus fibrosis of the intervertebral disc. At the level of the midvertebral body, there is a midline, sagittally oriented septum (meningovertebral ligament) anchoring the posterior longitudinal ligament to the periosteum, effectively dividing the anterior epidural space into 2 discrete compartments to the right and left of the midline septum (Fig 1). ${ }^{6,7}$ The anatomy and composition of the multiple meningovertebral ligaments in both the anterior and posterior epidural spinal compartments were well-delineated in a study 
using data from in vivo imaging, postmortem anatomy, and microscopy, in which they were found to comprise compact collagen attached to an area of thickened vertebral body periosteum. ${ }^{8}$ Geers et $\mathrm{al}^{7}$ demonstrated that the polygonal, stellar, or y-shaped deformation of the dural sac seen in patients with epidural lipomatosis is attributable to the presence of meningovertebral ligaments. These ligaments, and particularly the anterior meningovertebral ligament, are variably affected by pathology involving the epidural space. Kim et $\mathrm{al}^{9}$ demonstrated that whereas epidural hematoma and pyogenic infection disrupt the ventral meningovertebral ligament, a neoplasm leaves this anterior septum intact. The purpose of this study was to determine whether the identification of the ventral meningovertebral ligament on MR imaging can help differentiate between TB and pyogenic spondylodiscitis. It is thought that unlike pyogenic spondylodiscitis, TB spondylodiscitis spares the intervertebral disc in the setting of early infection because it lacks the proteolytic enzymes necessary to breach the disc. We hypothesize that similarly, TB spondylodiscitis will

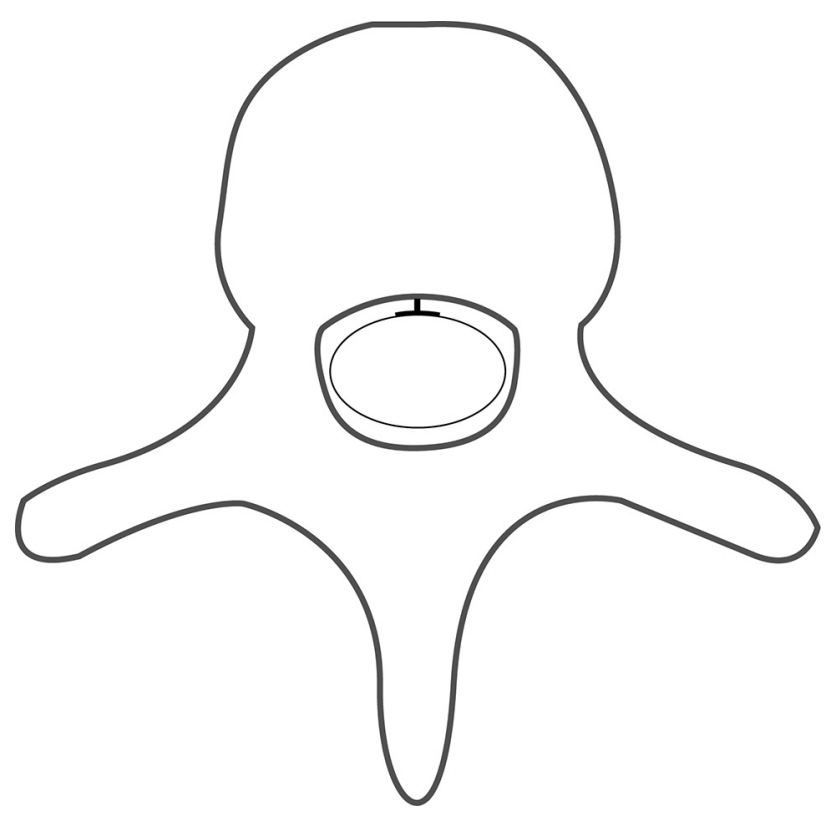

FIG 1. Graphic representation of normal anatomy. The anterior meningovertebral ligament anchors the periosteum to the posterior longitudinal ligament, effectively creating 2 distinct compartments. not affect the anterior meningovertebral ligament in the setting of anterior epidural abscess.

\section{MATERIALS AND METHODS}

This Health Insurance Portability and Accountability Act-compliant retrospective cohort study was performed with approval of the institutional review board of Montefiore Medical Center. We identified all patients who had MR imaging of the cervical, thoracic, or lumbar spine between May 2014 and September 2019 performed in the inpatient or emergency department setting who also had any acid-fast bacillus lab test performed (culture or stain, derived from abscess, body fluid, blood culture, bone marrow, CSF, respiratory, tissue, or wound) using the Looking Glass clinical analytics (Streamline Health, Atlanta, Georgia). Four hundred seventy-one patients were identified, of whom 85 had epidural abscess as per the radiology report. Final pathology and chart review were performed, and cases were further classified as either pyogenic or TB spondylodiscitis established by positive culture or gram stain. Cases were excluded if there was a dorsal epidural abscess alone without ventral involvement $(n=11)$, postoperative/ post traumatic infection $(n=5)$, no definite final diagnosis (patient lost to follow-up or died) $(n=3)$, no organism identified on culture $(n=14)$, phlegmon rather than organized abscess on imaging $(n=11)$, or final pathology demonstrated a noninfectious process $(n=1)$. The remaining 40 patients demonstrating a ventral epidural collection at the level of the vertebral body were included in the study. Demographics recorded included age, sex, and ethnicity and are provided in Table 1.

Spinal MRIs were acquired at multiple inpatient sites in our institution, using various $1.5 \mathrm{~T}$ and $3 \mathrm{~T}$ imaging systems including 1.5T Achieva, 1.5T Ingenia, 3T Intera, and 3T Ingenia MR imaging scanners (Philips Healthcare, Best, the Netherlands). Gadavist (gadobutrol) $0.1 \mathrm{mmol} / \mathrm{kg}$ (Bayer Schering Pharma, Berlin, Germany) intravenous contrast was administered. MR imaging sequences reviewed for each patient included axial and sagittal T2WI and T1WI with and without contrast. The imaging review was conducted by a team consisting of a Certificate of Added Qualification board-certified neuroradiologist (S.E.S. with 14 years' experience) and a fourth-year radiology resident (S.B.S. with 4 years' experience) using consensus review; a third Certificate of Added Qualification board-certified neuroradiologist (J.B. with 13 years' experience) adjudicated the differences when consensus could not be reached. The third reviewer was necessary in 3 cases. All reviewers were blinded to the final diagnosis. A unanimous determination was made regarding whether the anterior meningovertebral ligament was intact versus violated.

An intact anterior meningovertebral ligament was defined as an epidural abscess at the level of the vertebral body with an umbilicated, bilobed, or unilobed appearance (Figs 2 and 3), whereas a destroyed ligament was defined as a collection along the posterior aspect of the vertebral body with a

${ }^{a}$ For categoric variables, the frequency (No.) and percentage are reported. For continuous variables, the mean (SD) are reported. 

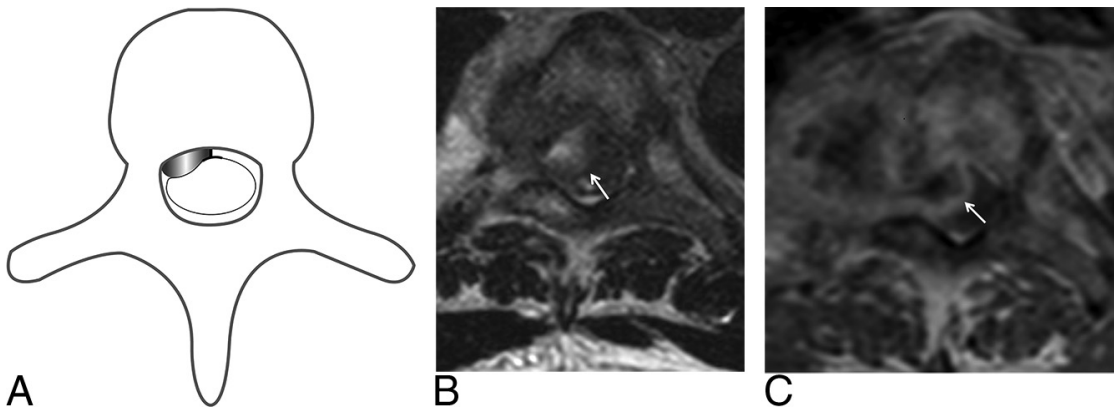

FIG 2. Preserved anterior meningovertebral ligament with a unilobed anterior epidural abscess. Graphic representation of a unilobed anterior epidural space abscess (A). Axial T2WI (B) and contrast-enhanced TIWI (C) show a unilobed appearance of the epidural abscess.

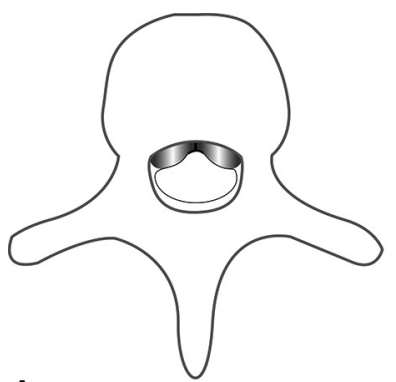

A

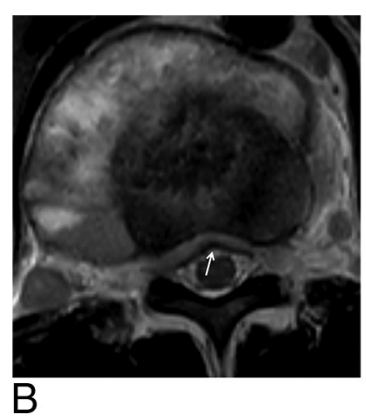

$\bar{B}$

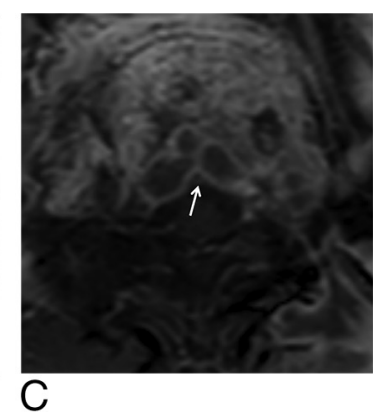

FIG 3. Preserved anterior meningovertebral ligament in 2 patients with bilobed anterior epidural abscess. Graphic representation of a bilobed anterior epidural space abscess (A). Axial T2WI $(B)$ and contrast-enhanced TIWI (C) show a bilobed appearance of the epidural abscess in the setting of a preserved anterior meningovertebral ligament (arrow).

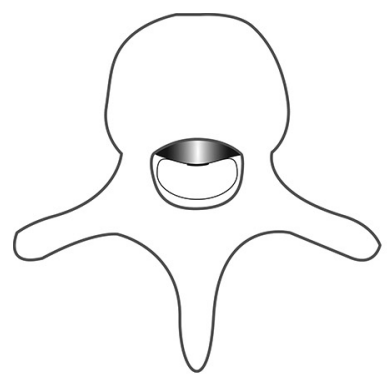

A

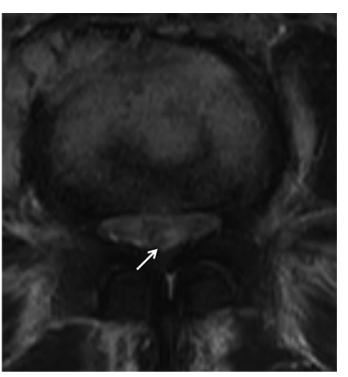

$\mathrm{B}$

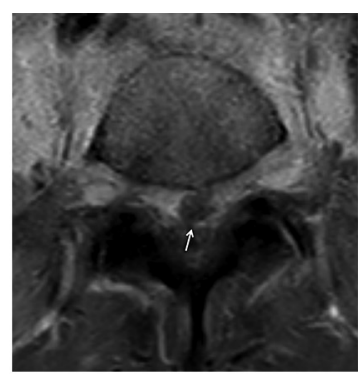

C
FIG 4. Destruction of the anterior meningovertebral ligament in 2 patients. Graphic representation of destruction of the anterior meningovertebal ligament $(A)$. Axial T2WI (B) demonstrates destruction of the anterior meningovertebral ligament (arrow). Axial contrast-enhanced TTWI (C) in a different patient similarly demonstrates destruction of the anterior meningovertebral ligament (arrow). The apices of the collections are midline and convex and do not respect left-right boundaries. independence was used to assess significant difference in race/ethnicity between the 2 groups and segmental location of epidural abscess. Independent 2-sample $t$ tests were performed to test for significant differences between the 2 groups in age. Sensitivity, specificity, and positive and negative predictive values were determined, and their confidence intervals were calculated using the Jeffreys method with EpiTools epidemiological calculators (2019 Ausvet; http://epitools.ausvet.com.au/content. php?page=home)

\section{RESULTS}

The demographic features of patients with TB spondylodiscitis and pyogenic spondylodiscitis are shown in Table 1. There were no significant differences between the 2 groups in age, sex, race/ ethnicity, or segmental location of anterior epidural abscess. All patients included in the study had laboratory confirmation of the pathogen. Eighteen of 35 (51\%) patients classified as having pyogenic osteomyelitis had laboratory confirmation of the pathogen based on biopsy, and 17/35 (49\%) patients classified as having pyogenic osteomyelitis had confirmation of the pathogen based on positive blood cultures. The biopsy results are summarized in Table 2 several patients had $>1$ pathogen isolated. Of note, one of the patients with TB spondylodiscitis had 2 separate abscesses at remote spinal levels, which were counted as 2 different abscesses.

Prevalence of imaging features associated with tuberculous and pyogenic anterior epidural abscesses in the studied cohort are detailed in Table 3. There was a significant differ- dorsally convex midline apex and direct communication between the right and left compartments of the epidural space (Fig 4). Thus, the anterior meningovertebral ligament was not directly visualized, but rather, its status was inferred on the basis of the shape of the anterior epidural abscess. In addition, the segmental location of the anterior epidural abscess (cervical, thoracic, or lumbar) was recorded.

Statistical analyses were performed using SPSS (Versions 24 and 25; IBM, Armonk, New York). Demographic and imaging characteristics were compared between the groups with TB spondylodiscitis and pyogenic spondylodiscitis. A $\chi^{2}$ test of ence between the 2 groups in terms of the appearance of the meningovertebral ligament, with $5 / 6(83.3 \%)$ cases of tuberculous-associated anterior epidural abscess demonstrating an intact meningovertebral ligament, and 0/35 (0\%) cases with pyogenic osteomyelitis-associated infection demonstrating an intact meningovertebral ligament $(P<.001)$. The single case of tuberculosis with a destroyed meningovertebral ligament also demonstrated extensive vertebral body collapse (75\% loss of vertebral body height). The presence of an intact anterior meningovertebral ligament had $83.3 \%$ sensitivity (95\% confidence interval, $44.2 \%-98.1 \%$ ), $100 \%$ specificity ( $95 \%$ confidence interval, 
Table 2: Pyogenic Biopsy and Culture Results

\begin{tabular}{lc}
\multicolumn{1}{c}{ Organisms Isolated } & No. \\
\hline Staphylococcus species & 23 \\
Streptococcus species & 6 \\
Escherichia coli & 2 \\
Other: Klebsiella, Bacillus cereus, Proteus, Hemophilus & 8 \\
$\quad$ species, Enterococcus, Enterobacter & \\
\hline
\end{tabular}

\begin{tabular}{|c|c|c|c|}
\hline & $\begin{array}{l}\text { M Tuberculosis } \\
(n=6)\end{array}$ & $\begin{array}{l}\text { Pyogenic } \\
(n=35)\end{array}$ & $\begin{array}{c}P \\
\text { Value }\end{array}$ \\
\hline Anterior septum violated & $1(16.7 \%)$ & 35 (100\%) & $<.001$ \\
\hline Location & & & .90 \\
\hline Cervical & 0 & $5(14.3 \%)$ & \\
\hline Thoracic & $4(66.7 \%)$ & $8(22.9 \%)$ & \\
\hline Lumbar & $1(16.7 \%)$ & $19(54.3 \%)$ & \\
\hline Thoracolumbar & $1(16.7 \%)$ & $1(2.9 \%)$ & \\
\hline Lumbosacral & 0 & $2(5.7 \%)$ & \\
\hline
\end{tabular}

93\%-100\%), 100\% positive predictive value (95\% confidence interval, $62.1 \%-1 \%$ ), and $97.2 \%$ negative predictive value $(95 \%$ confidence interval, $88 \%-1 \%$ ) for TB spondylodiscitis.

\section{DISCUSSION}

Mycobacterium tuberculosis is a Gram-positive acid-fast bacillus, which can primarily infect bone or secondarily spread to the axial skeleton via the Batson venous plexus from remote sites of disease. Several patterns of vertebral body infection are described in $M$ tuberculosis spondylodiscitis, including central, anterior, posterior, and paradiscal. Tuberculosis occurs more often in the thoracic spine, followed by the lumbar spine. ${ }^{10}$ Late in the disease, osteolysis results from bone necrosis, causing spinal instability and the typical gibbus deformity to develop. ${ }^{10}$ Epidural abscess may be present in TB spondylodiscitis, particularly in association with paradiscal infection and can be difficult to differentiate from pyogenic osteomyelitis in the absence of other stereotypical features. $^{10}$

Differentiation between pyogenic and TB spondylodiscitis is often a diagnostic challenge because few imaging features are shown to reliably distinguish the 2 entities. Moreover, approximately $43 \%-70 \%$ of percutaneous biopsies are culture-negative in the setting of pyogenic and TB spondylodiscitis, even with high clinical and radiologic suspicion, with only marginally improved percentages reported for open biopsies. ${ }^{11,12}$ In this study, we found that preservation of the anterior meningovertebral ligament had high sensitivity $(83.3 \%)$ and specificity (100\%) for the diagnosis of tuberculous infection in the setting of anterior epidural abscess, though these results must be interpreted with some degree of caution given the small sample size. The single case of culture-positive TB infection with a destroyed anterior meningovertebral ligament also demonstrated extensive vertebral body height loss (75\% loss of vertebral body height). We postulate that the vertebral body damage itself was likely responsible for injury to the anterior meningovertebral ligament, rather than primary destruction due to the infection.
Several prior studies have examined imaging features that differentiate pyogenic from TB spondylodiscitis. Features favoring TB infection over typical pyogenic infection include intervertebral disc space sparing, posterior element involvement, extensive paraspinal soft-tissue involvement, multiple vertebral body involvement, a heterogeneous vertebral body enhancement pattern, and subligamentous spread. ${ }^{1,5,13,14}$ The status of the anterior meningovertebral ligament represents a novel addition to the existing body of literature and may serve as collateral information in cases in which both diagnoses are being entertained.

The anterior meningovertebral ligament has been shown to have an impact on the spread of disease in the context of noninfectious pathology. In the setting of degenerative disease, the anterior meningovertebral ligament limits transverse migration of extruded disc material. ${ }^{8}$ Schellinger et $\mathrm{al}^{6}$ initially described progressive destruction of this midline septum in the setting of infection and neoplasm, and Kim et $\mathrm{al}^{9}$ showed that a unilobed or bilobed appearance of a ventral epidural collection had a 76\%-82\% sensitivity and $73 \%-80 \%$ specificity in identifying neoplastic compared with nonneoplastic anterior epidural space lesions, including hematoma and infection. Hong et $\mathrm{al}^{15}$ noted that TB associated anterior paraspinal abscess of the thoracic spine will not encase the intercostal arteries due to an inability to breach the anterior longitudinal ligament. In the current study, the observed destruction of the anterior midline meningovertebral ligament in the setting of pyogenic spondylodiscitis is consistent with that previously reported; the observed preservation of the ligament in TB suggests that mycobacterial infection behaves more similar to neoplasm than to trauma or bacterial infection.

The pathophysiologic basis of the observed effects on the anterior meningovertebral ligament is likely related to the unique features of the microorganism. An explanation commonly given for the observed sparing of the intervertebral disc during early stages of infection is that TB characteristically lacks proteolytic enzymes. ${ }^{16-18}$ Additionally, as an aerobic organism, $M$ tuberculosis requires greater arterial supply to survive and is therefore more slow-growing than other infectious organisms, ${ }^{19}$ taking up to 11.2 months before clinical signs present, compared with 6.4 months in patients with pyogenic spondylodiscitis. ${ }^{1}$ Lack of proteolytic enzymes is an important factor that may explain why $M$ tuberculosis infection typically spares the anterior meningovertebral ligament. Although the meningovertebral ligament varies in thickness between different spinal segments, increasing in thickness at the level of the lumbar spine, we found no significant difference between the pyogenic and TB cases in terms of segmental involvement.

There are several limitations to this study. First, the sample size is limited, with only $15 \%$ of total cases positive for tuberculosis. However, this limitation reflects the relatively low prevalence of tuberculosis in the studied population. The relatively high number of pyogenic cases improves the specificity and negative predictive value of this finding, while reducing the sensitivity and positive predictive value. Of note, the search criteria used to develop the cohort included only patients who underwent acid-fast bacillus testing. Therefore, while we did not capture all epidural abscesses in our institution during the study period; the study specifically addresses those cases in which both pyogenic and TB 
infections were differential considerations and in which a radiologic sign might be of greatest utility in discriminating the 2 organisms. Finally, although all studies were performed at the same institution, not all studies were performed on the same scanner or with identical parameters, and the variability in image quality may potentially impact interpretation.

\section{CONCLUSIONS}

The presence of an intact anterior meningovertebral ligament on spinal MR imaging has high specificity, sensitivity, and negative predictive value for the diagnosis of TB spondylodiscitis, though these results remain to be validated in a larger sample. Attention to this subtle imaging feature might facilitate early pathogendirected treatment and ultimately reduce morbidity and the length of stay in the setting of otherwise inconclusive clinical parameters.

\section{ACKNOWLEDGMENTS}

We thank Dr Victoria Chernyak for her contributions to data mining and Dr Kenny Ye for his assistance with statistical analysis.

\section{REFERENCES}

1. Lee KY. Comparison of pyogenic spondylitis and tuberculous spondylitis. Asian Spine J 2014;8:216-23 CrossRef Medline

2. Kumar Y, Gupta N, Chhabra A, et al. Magnetic resonance imaging of bacterial and tuberculous spondylodiscitis with associated complications and non-infectious spinal pathology mimicking infections: a pictorial review. BMC Musculoskelet Disord 2017;18:244 CrossRef Medline

3. Darouiche RO. Spinal epidural abscess. N Engl J Med 2006;355:201220 CrossRef Medline

4. De la Garza Ramos R, Goodwin CR, Abu-Bonsrah N, et al. The epidemiology of spinal tuberculosis in the United States: an analysis of 2002-2011 data. J Neurosurg Spine. 2017;26:507-12 CrossRef Medline

5. Jung NY, Jee WH, Ha KY, et al. Discrimination of tuberculous spondylitis from pyogenic spondylitis on MRI. AJR Am J Roentgenol 2004;182:1405-10 CrossRef Medline
6. Schellinger D, Manz H, Vidic B, et al. Disk fragment migration. Radiology 1990;175:831-36 CrossRef Medline

7. Geers C, Lecouvet FE, Behets C, et al. Polygonal deformation of the dural sac in lumbar epidural lipomatosis: anatomic explanation by the presence of meningovertebral ligaments. AJNR Am J Neuroradiol 2003;24:1276-82 Medline

8. Scapinelli R. Anatomical and radiologic studies on the lumbosacral meningo-vertebral ligaments of humans. J Spinal Disord 1990;3:615 CrossRef Medline

9. Kim DH, Rosenblum JK, Panghaal VS, et al. Differentiating neoplastic from nonneoplastic processes in the anterior extradural space. Radiology 2011;260:825-30 CrossRef Medline

10. Rivas-Garcia A, Sarria-Estrada S, Torrents-Odin C, et al. Imaging findings of Pott's disease. Eur Spine J 2013;22:567-78 CrossRef Medline

11. Colmenero J, Jimenez-Mejias M, Reguera J, et al. Tuberculous vertebral osteomyelitis in the new millennium: still a diagnostic and therapeutic challenge. Eur J Clin Microbiol Infect Dis 2004;23:477-83 CrossRef Medline

12. Gras G, Buzele R, Parienti J, et al. Microbiological diagnosis of vertebral osteomyelitis: relevance of second percutaneous biopsy following initial negative biopsy and limited yield of post-biopsy blood cultures. Eur J Clin Microbiol Infect Dis 2014;33:371-75 CrossRef Medline

13. Arizono T, Oga M, Shiota E, et al. Differentiation of vertebral osteomyelitis and tuberculous spondylitis by magnetic resonance imaging. Int Orthop 1995;19:319-22 CrossRef Medline

14. Frel M, Białecki J, Wieczorek J, et al. Magnetic resonance imaging in differentatial diagnosis of pyogenic spondylodiscitis and tuberculous spondylodiscitis. Pol J Radiol 2017;82:71 CrossRef Medline

15. Hong SH, Choi JY, Lee JW, et al. MR imaging assessment of the spine: infection or an imitation? Radiographics 2009;29:599-612 CrossRef Medline

16. Chang MC, Wu HT, Lee CH, et al. Tuberculous spondylitis and pyogenic spondylitis: comparative magnetic resonance imaging features. Spine 2006;31:782-88 CrossRef Medline

17. Smith AS, Weinstein MA, Mizushima A, et al. MR imaging characteristics of tuberculous spondylitis vs vertebral osteomyelitis. $A J R$ Am J Roentgenol 1989;153:399-405 CrossRef Medline

18. Almeida Á. Tuberculosis of the spine and spinal cord. Eur J Radiol 2005;55:193-201 CrossRef Medline

19. Moorthy S, Prabhu NK. Spectrum of MR imaging findings in spinal tuberculosis. AJR Am J Roentgenol 2002;179:979-83 CrossRef Medline 